\title{
Elastic incoherent neutron scattering as a probe of high pressure induced changes in protein flexibility
}

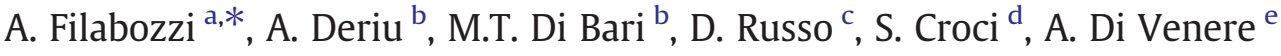 \\ a Università di Roma "Tor Vergata", Dipartimento di Fisica and CNISM, Roma, Italy \\ b Università di Parma, Dipartimento di Fisica and CNISM, , Parma, Italy \\ c CNR-INFM, CRS-SOFT, Institut Laue-Langevin, Grenoble, France \\ ' Università di Parma, Dipartimento di Sanità Pubblica, CNISM and INBB, Parma, Italy \\ e Università di Roma "Tor Vergata", Dipartimento di Medicina Sperimentale e Scienze Biochimiche, Roma, Italy
}

\section{A R T I C L E I N F O}

\section{Article history:}

Received 27 March 2009

Received in revised form 26 August 2009

Accepted 31 August 2009

Available online 6 September 2009

\section{Keywords:}

Neutron scattering

High pressure

Protein unfolding

Protein flexibility

Trypsin

Lysozyme

Beta-lactoglobulin

\begin{abstract}
A B S T R A C T
We report here the results of elastic incoherent neutron scattering experiments on three globular proteins (trypsin, lysozyme and $\beta$-lactoglobulin) in different pressure intervals ranging from 1 bar to $5.5 \mathrm{kbar}$. A decrease of the mean square hydrogen fluctuations, $\left\langle u^{2}\right\rangle$, has been observed upon increasing pressure. Trypsin and $\beta$-lactoglobulin behave similarly while lysozyme shows much larger changes in $\left\langle u^{2}\right\rangle$. This can be related to different steps in the denaturing processes and to the high propensity of lysozyme to form amyloids. Elastic incoherent neutron scattering has proven to be an effective microscopic technique for the investigation of pressure induced changes in protein flexibility.
\end{abstract}

(c) 2009 Elsevier B.V. All rights reserved.

\section{Introduction}

Conformational changes play a major role in the regulation of the function and biological activity of proteins; they affect also global folding/unfolding transitions. During denaturation, unfolding processes often lead to the formation of protein aggregates. In the past few years these aggregates have been studied in some detail since they have been found to behave as a marker in many diseases (e.g. Alzheimer's, prion, Parkinson's, lysozyme amyloidosis) [1]. Aggregation is induced by the onset of partially unfolded conformations. These intermediate states have been observed during pressure- and colddenaturation since these processes have a milder action with respect to other methods such as chemical and thermal denaturation. For instance, during lysozyme cold denaturation, intermediates with secondary or higher order structures similar to those observed in early folding process, are formed [2].

The study of protein unfolding or aggregation pathways may become complex owing to the fact that different denaturing agents lead to different end points. Moreover, it is difficult to discriminate if a particular structural state of a protein is due to its intrinsic stabilizing

\footnotetext{
* Corresponding author. Università di Roma "Tor Vergata", Dipartimento di Fisica, Via Ricerca Scientifica 1, I-00133 Roma, Italy. Fax: +3906 2023507.

E-mail address: Alessandra.Filabozzi@roma2.infn.it (A. Filabozzi).
}

factors or to the binding with the denaturant molecules. In this respect high hydrostatic pressure is a very effective tool to perturb protein conformations as documented by several authors [3-5].

A recent investigation on the microscopic physical mechanisms promoting cold denaturation indicates that they are similar to those acting in pressure-induced denaturation. In both cases water molecules move to the interior of the protein destabilizing hydrophobic contacts and creating different solvent-separated configurations [6].

Contrary to heat-denaturation, which is controlled by entropy increase, high-pressure denaturation is driven by a decrease in volume induced by the reduction of intra-molecular voids or to the enhancement of protein-solvent interactions [7]. In this frame hydrophobic interactions, that stabilize tertiary and quaternary protein structures, play a crucial role. Neutron elastic and inelastic scattering are very effective techniques to investigate different aspects of biomolecular dynamics; in proteins they provide a direct way to probe specific motions of the polypeptide backbone and of associated hydration water, and to measure flexibility changes connected with unfolding and aggregation. In biomolecules incoherent scattering dominates due to the high number of hydrogens present and to their large incoherent scattering cross section. Moreover the solvent contribution can be minimized using deuterated solvents since the scattering cross section of deuterium is twenty times lower than that of hydrogen. In previous neutron quasielastic 
scattering (QENS) we have investigated the pressure dependence of the local internal dynamics in two monomeric globular proteins, trypsin and lysozyme, in a pressure range (1 to 1200 bar) which is well below the denaturation threshold [8,9]. Recently further QENS studies and MD simulations on lysozyme in a similar pressure range have also been published [10-12].

In the past few years it has been shown that incoherent elastic neutron scattering (ENS), provide information on the average hydrogen mean square fluctuations, $\left\langle u^{2}\right\rangle$ that can be related to the average interatomic force constants and to the thermodynamic resilience of the molecular system [13-15].

In this work we focus on the application of ENS to the investigation of the dynamical changes induced by high pressure on three small monomeric globular proteins: trypsin, lysozyme and $\beta$-lactoglobulin. They have all been already studied in detail by other spectroscopic techniques in the pressure range from ambient to the denaturation limit: their conformational changes turn out to be quite different, showing a complex pressure dependence. They allow us to investigate several aspects of protein behavior.

In the case of trypsin we have investigated protein flexibility in its predenaturational state. At low pressure values (below 1 kbar) the protein is still active and then, when pressure is further raised, it starts to unfold towards a stable intermediate state $[16,17]$. On the contrary, in the case of lysozyme pressure immediately inactivates the protein and the partial unfolded state found at $5.5 \mathrm{kbar}$ is inclined to aggregate $[18,19]$. Finally for $\beta$-lactoglobulin we have studied the reversible denaturation process taking place in the range 1-1000 bar.

\section{Materials and methods}

\subsection{Sample preparation}

Three protein samples have been considered: trypsin (23.5 kDa) from bovine pancreas, lysozyme $(14.4 \mathrm{kDa})$ and $\beta$-lactoglobulin $(18.4 \mathrm{kDa})$. They were purchased from SIGMA Chemical Co in fully hydrated form and were employed without further purification. The proteins were dissolved in pure $\mathrm{D}_{2} \mathrm{O}$ solvent in order to exchange labile hydrogen atoms, and then lyophilized. Solution samples were obtained by dissolving the protein powder in $\mathrm{D}_{2} \mathrm{O}$ buffer with final concentration of $100 \mathrm{mg} / \mathrm{ml}$ (indicated as $10 \%$ solution in the following).

For the trypsin solution pD was adjusted to 3 by adding a small amount of $\mathrm{HCl}(1 \mathrm{mM})$. For lysozyme, two distinct buffers were used: a $10-\mathrm{mM}$ tris buffer with a final $\mathrm{pD}=6.0$ and a $50-\mathrm{mM}$ acetate buffer with a final $\mathrm{pD}=5$; in both conditions it is known that the protein keeps its monomeric structure [20]. The lysozyme total charge was around +9 . For $\beta$-lactoglobulin a $50-\mathrm{mM}$ tris buffer was used as well, adjusted with $\mathrm{HCl}$ to a final $\mathrm{pD}=7.9$. Protein solutions have been finally stirred for $10 \mathrm{~min}$ at $9000 \mathrm{r} / \mathrm{min}$ at $292 \mathrm{~K}$.

\subsection{Fluorescence and circular dicroism analysis}

Circular dicroism spectra, performed in the UV region on lysozyme and trypsin solutions before and after the pressure scans, revealed that the secondary structure of the proteins was substantially unchanged and did not suffer from any modification owing to the applied pressure (data not shown).

Changes in proteins tertiary and/or quaternary structure have been also checked by fluorescence spectroscopy. Lysozyme fluorescence light scattering measurements under pressure were performed using a ISS (Champaign, IL, USA) spectrofluorometer equipped with a pressure cell as described by Paladini and Weber [21]. Right angle light scattering was measured at $520 \mathrm{~nm}$ with $8 \mathrm{~nm}$ band passes for sample excitation and light emission collection. The fluorescence spectra collected at each pressure value have been corrected measuring the scattering of buffer solution in the same condition.
The samples were thermostated at $293 \mathrm{~K}$ by an external bath circulator.

In the case of trypsin, we have monitored the fluorescence signal of 1-anilino-8-naphthalene-sulfonic acid (ANS, Molecular Probe, INC.), a fluorescent probe that changes its emission intensity upon binding non-covalently to protein hydrophobic regions. In this context the ANS fluorescence signal should increase upon protein aggregation.

\subsection{High-pressure neutron cells}

Two different experimental setups have been adopted with different high-pressure cells, one for the trypsin experiments and a second one for both lysozyme and $\beta$-lactoglobulin. In the first case the cell consists of a thin-wall stainless-steel capillary tube (inner diameter $1 \mathrm{~mm}$, wall thickness $0.2 \mathrm{~mm}$, measured transmission $\sim 83 \%$ ), arranged in a concertina-like shape. A temperature control system with temperature stability $\pm 0.5 \mathrm{~K}$ was used. For lysozyme and $\beta$-lactoglobulin, a high pressure system developed at the ILL within the IN13-CRG has been used. This cell, shown in Fig. 1, is made up from a stainless steel capillary $(0.5$ and $1.6 \mathrm{~mm}$ inner and outer diameters), with a cylindrical geometry (outer diameter $d=36 \mathrm{~mm}$, height $H=50 \mathrm{~mm}$ ). Using $2.23 \AA$ neutrons the empty cell had a measured transmission of $\sim 75 \%$ while the cell filled with the protein solution had a transmission of about $72 \%$. The scattering contributions of the cell and of the buffer have been already analyzed in a previous paper [9] that reports the results a QENS experiment with a resolution similar to that of the previous one. The reader may refer to that paper for more details.

The high pressure apparatus (manufactured by Top Industrie, S.A., France) was composed by a primary pressure hand pump (max $P=700$ bar), a pressure intensifier ( $\max P=7 \mathrm{kbar}$ ), pressure gauges (pressure accuracy \pm 10 bar), stainless steel fittings and valves.

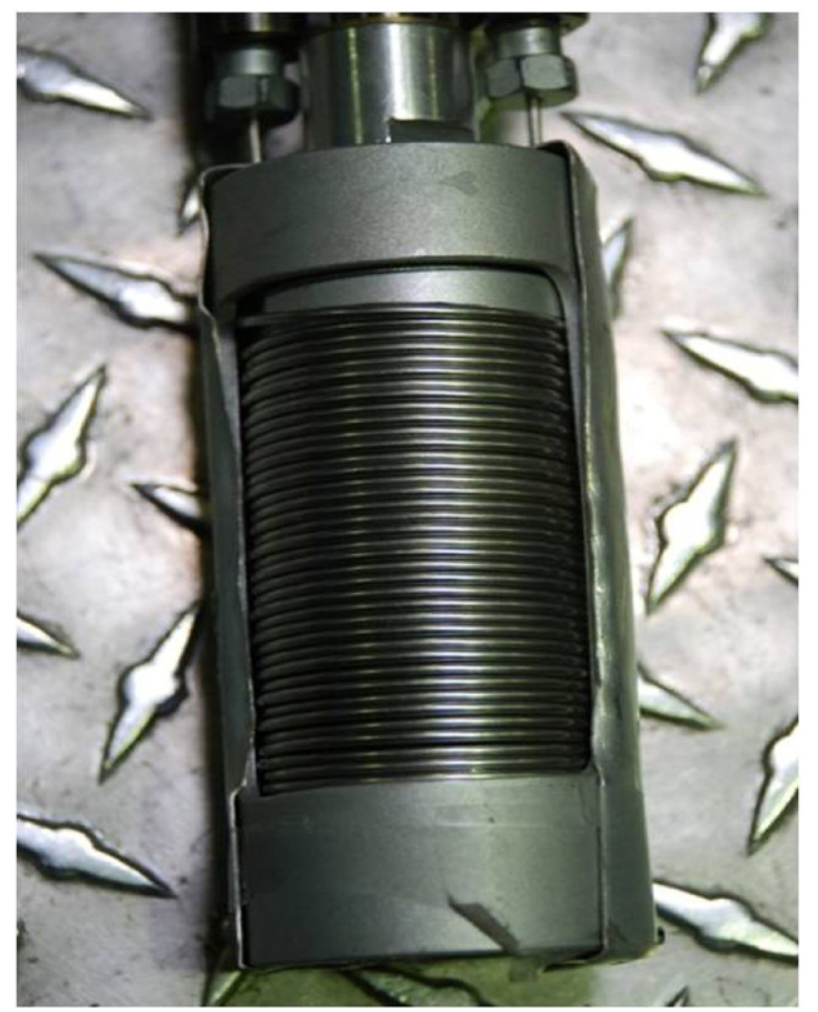

Fig. 1. High pressure cylindrical cell made from an inox capillary tube $\left(d_{\text {int }}=0.5 \mathrm{~mm}\right.$, $\left.d_{\text {ext }}=1.6 \mathrm{~mm}\right)$ enrolled into an anular geometry $(H=50 \mathrm{~mm} ; D=36 \mathrm{~mm})$. The sample volume that can be illuminated by neutron beam is $1.3 \mathrm{~cm}^{3}$. The empty cell transmission is 0.75 at $2.23 \AA$. The cell has been tested to work up to $7 \mathrm{kbar}$. 


\subsection{Elastic neutron scattering experiments}

Incoherent elastic neutron scans were performed using IN13 at the ILL (Grenoble, Fr). IN13 is a thermal $(\lambda=2.23 \AA$ ) backscattering spectrometer that provides a relatively high $Q$-range (up to about $4.6 \AA^{-1}$ ) with an almost $Q$-independent energy resolution $(\triangle E \sim 8 \mu \mathrm{eV}$, FWHM) [22]. This corresponds to a space scale of about $1-20 \AA$ and to a time window that includes all motions slower than $\sim 80 \mathrm{ps}$. In the slab geometry, adopted in the case of the experiments on trypsin, the sample angle with respect to the incoming neutron beam was $45^{\circ}$. The acquisition time for each run was about 8 hours. Vanadium standard (a $2 \mathrm{~mm}$ thick slab and a cylindrically wrapped foil), empty cell, and pure buffer runs, were also performed for data normalization and correction. The thermodynamic conditions for the three protein solutions were the following:

trypsin: five different temperatures from 275 to $318 \mathrm{~K}$; pressure from 1 to 2 kbar;

lysozyme (tris buffer): constant temperature (298 K); pressure cycle: 1 bar , 3.5 kbar, 5.5 kbar, 1 bar;

lysozyme (acetate buffer): constant temperature (298 K); pressure cycle: 1 bar , 1 kbar, 2 kbar, 1 bar;

$\beta$-lactoglobulin: constant temperature (298 K); pressure cycle: 1 bar , 1 kbar, 1 bar;

Two different experiments on IN13 have been performed, one for trypsin and another one for $\beta$-lactoglobulin and lysozyme.

\subsection{Neutron data analysis}

The elastic scattering intensities were corrected for detector efficiency using a Vanadium run, for transmission and geometry effects and for the empty cell and buffer contributions using standard ILL routines [23]. They were then normalized to the elastic scattering intensity of a "reference" sample: i.e. the same protein solution at ambient conditions: $T_{0}=298 \mathrm{~K}$ and $P_{0}=1$ bar [12].

The normalized incoherent elastic scattering intensity, $I_{\mathrm{el}}(Q)$, was analyzed in the Gaussian approximation. In this frame, at $Q$-values below $\sim 2 \mathrm{~A}^{-1}$, the mean square atomic fluctuations can be described in terms of a Lamb-Mössbauer like factor:

$I_{\mathrm{el}}(Q)=I_{\mathrm{el}}(Q, \omega=0)=\exp \left(-Q^{2}\left\langle u^{2}\right\rangle\right) / 3$

where $\left\langle u^{2}\right\rangle$ represents the hydrogen mean square fluctuations (MSF) at the given $T$ and $P$. With the normalization adopted for lysozyme and $\beta$-lactoglobulin, the difference mean square fluctuations $\Delta\left\langle u^{2}\right\rangle$ (DMSF) with respect to the reference sample is directly derived from the fit as:

$\Delta\left\langle u^{2}\right\rangle=\left\langle u^{2}(T, P)\right\rangle-\left\langle u^{2}\left(T_{0}, P_{0}\right)\right\rangle$

The use of DMSF makes it possible to evidence small relative differences in the atomic fluctuations. In the following, the slope, $\left\langle x^{2}\right\rangle$, of $\ln \left(I_{\mathrm{el}}\right)$ vs. $Q^{2}$ is considered. We have then: $\left\langle u^{2}\right\rangle / 3=\left\langle x^{2}\right\rangle$ or $\Delta\left\langle u^{2}\right\rangle /$ $3=\Delta x^{2}$.

\section{Results and discussion}

\subsection{Trypsin}

Up to date, several studies have been performed on trypsin to monitor the effects of pressure on its enzymatic activity. Far beyond its denaturation limit, trypsin shows an increase of the enzymatic activity up to 600 bar at $293 \mathrm{~K}[17,24]$. Recently Ruan et al. [16,24] have evidenced high-pressure changes in the trypsin tertiary structure: they were able to isolate a stable partly denatured state at $6.5 \mathrm{kbar}$.

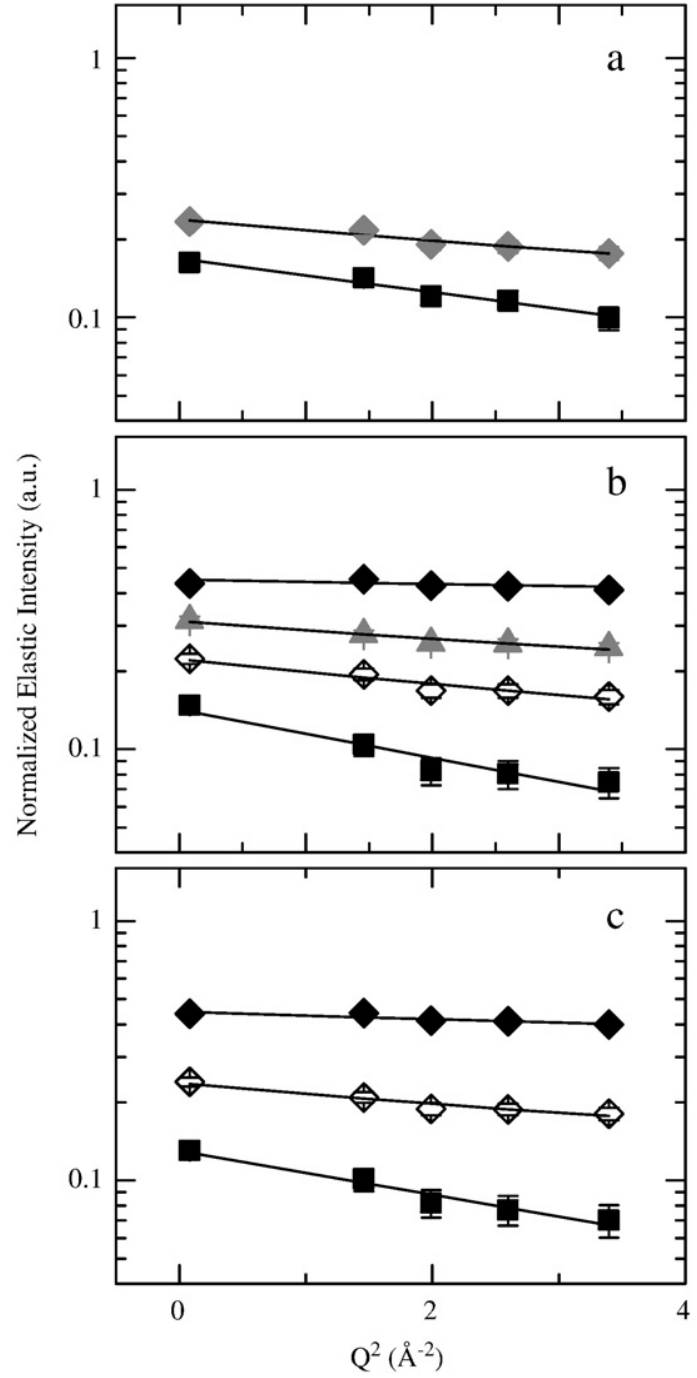

Fig. 2. Elastic scattering intesities vs. $Q^{2}$ for trypsin, at different termodynamic conditions. (a) $T=298 \mathrm{~K}, p=1$ bar (black squares) and $p=1330$ bar (grey diamonds); (b) $T=308 \mathrm{~K}, p=1$ bar (black squares), $p=540$ bar (open diamonds), $p=770$ bar (grey triangles), $p=1440$ bar (black diamonds); (c) $T=318 \mathrm{~K}, p=1$ bar (black squares), $p=570$ bar (open diamonds), $p=1490$ bar (black diamonds). In each panel the data have been shifted vertically with respect to each other for better visibility. The straight lines are fits to Eq. (1).

As an example, in Fig. 2 the normalized elastic scattering intensities are shown vs. $Q^{2}$ for trypsin at different temperatures and pressures. From the fit of these data to Eq. 1, the mean square hydrogen fluctuations have been derived. In Fig. 3 we report the difference slope $\Delta x^{2}$ for trypsin at different temperatures (between 275 and $318 \mathrm{~K}$ ) as a function of pressure (in a range between 1 and 2000 bar). We observe a similar behavior at all the investigated temperatures. The measured mean square fluctuation is constant within the experimental accuracy up to $p \approx 1300$ bar. This indicates that protein flexibility is not modified appreciably in this range of temperature/ pressure. This agrees with the fact that protein activity is not inhibited in this pressure range [17]. On the contrary, at $p>1300$ bar a significant decrease in the MSF is observed. This suggests a loss of protein flexibility induced by a decrease in volume due to the reduction of internal cavities and voids and/or by modifications of the surrounding solvent. The synergy of high pressure and high temperature drives the protein towards a partially unfolded state, as found by Ruan et al. [16] in analogous conditions.

Changes in the protein quaternary structure (i.e. aggregation) have been ruled out on the basis of the signal of the fluorescent probe ANS 


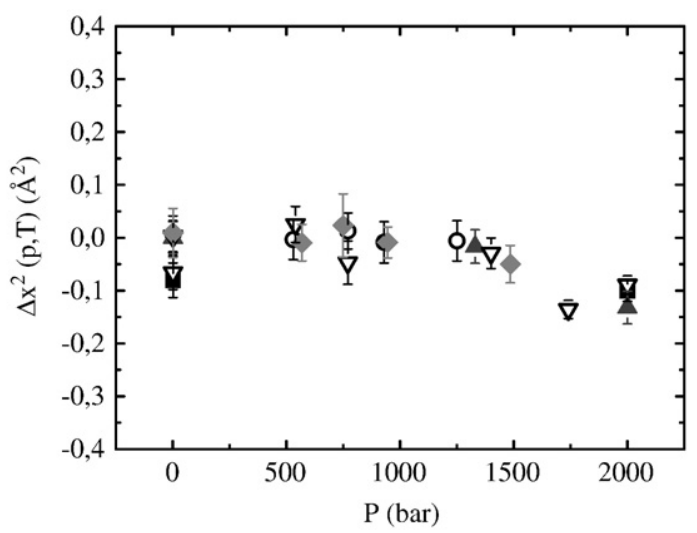

Fig. 3. Pressure dependence of the difference slope $\Delta x^{2}$, that is $\Delta\left\langle u^{2}(P, T)\right\rangle / 3$, for trypsin, at different temperatures (black squares: $T=279 \mathrm{~K}$, open circles: $283 \mathrm{~K}$, grey triangles: $298 \mathrm{~K}$, open triangles: $308 \mathrm{~K}$, grey diamonds: $318 \mathrm{~K}$ ). Normalization has been performed with respect to the data measured at $P_{0}=1$ bar and $T_{0}=298 \mathrm{~K}$.

bound to the protein. The ANS spectra recorded form 1 to $1000 \mathrm{bar}$ have very similar shape and intensity. This indicates that no appreciable aggregation occurs with increasing pressure, even though some differences may occur at the protein tertiary structure without involving changes in the molecular weight.

\section{2. $\beta$-lactoglobulin}

$\beta$-lactoglobulin is a quite compact protein composed essentially of a beta-barrel core. Upon increasing pressure up to 1000 bar (Fig. 4) the changes in the difference mean square fluctuations, DMSF, are similar to those observed in trypsin. This suggests that the observed effects do not depend on the tertiary structure of the protein. Moreover, following pressure release the protein mobility of the native state is re-acquired, suggesting a complete reversibility of the phenomenon. Therefore, at room temperature and in the applied pressure range, it can be concluded that the reduced mobility of the protein may be related to structural rearrangements of the hydration water shell. Upon raising pressure below the denaturation threshold we observe changes in protein flexibility that are related to the effect of applied pressure on the first/second hydration water layer (strengthening of hydrogen bond around hydrophilic sites, increase of local disorder around hydrophobic sites accompanied by a reduction of available volume for fluctuations). The picture that arises is that the "nativelike" protein upon increasing pressure, is in an environment more rigid than that at ambient pressure and consequently its fluctuations are slowed down and eventually inhibited. This effect can be compared to that of proteins in highly viscous solvents.

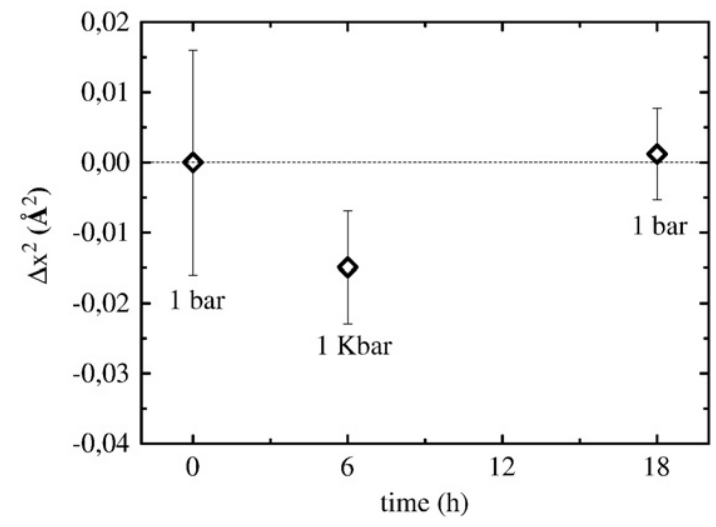

Fig. 4. Time evolution of the difference slope $\Delta x^{2}$, that is $\Delta\left\langle u^{2}(P, T)\right\rangle / 3$, measured for $\beta$ lactoglobulin. Normalization has been performed with respect to the data measured at $P_{0}=1$ bar and $T_{0}=298 \mathrm{~K}$.

\subsection{Lysozyme}

For lysozyme the effect of pressure on two different buffers has been investigated. As in the case of trypsin and of $\beta$-lactoglobulin, for lysozyme we observe a reduction of DMSF with increasing pressure. In Figs. $5 \mathrm{a}$ and $\mathrm{b}$ the time evolution of the difference slope $\Delta x^{2}$ is shown for the two solutions: lysozyme + tris buffer at $p=1,3500,5500,1$ bar (Fig. 5a); lysozyme + acetate buffer at $p=1,1000,2000,1$ bar (Fig. 5b). The DMSF increases by a factor of 3 in going from ambient pressure to 5.5 kbar. After releasing pressure, it decreases to a value slightly higher than that of the starting fresh solution. In this case the observed compaction, at such high pressure, may be ascribed to a compression of the hydrophobic core of the protein consisting of bulky side chains [18].

In Fig. 6 fluorescence light scattering intensity under pressure is shown for lysozyme dissolved in tris and acetate buffer, after subtraction of the buffer contribution. In the case of acetate, the large increase of the intensity with respect to tris, indicates that lysozyme is very sensitive to pressure and prone to aggregate at lower pressure values. This result correlates fairly well with the neutron scattering data reported in Fig. 5, where we can see that the same value of the slope $\Delta x^{2}$, obtained at $5.5 \mathrm{kbar}$ in tris, is reached, in the case of acetate, already at 2 kbar.

The fact that the slope values observed at $1 \mathrm{kbar}$ for lysozyme are quite different from those of trypsin and $\beta$-lactoglobulin $\left(\Delta x^{2} \approx 0.012 \AA^{2}\right.$ for trypsin and $\beta$-lactoglobulin, and almost 3 times higher for lysozyme) support the hypothesis that the observed phenomenon is more than a simple solvent rearrangement around
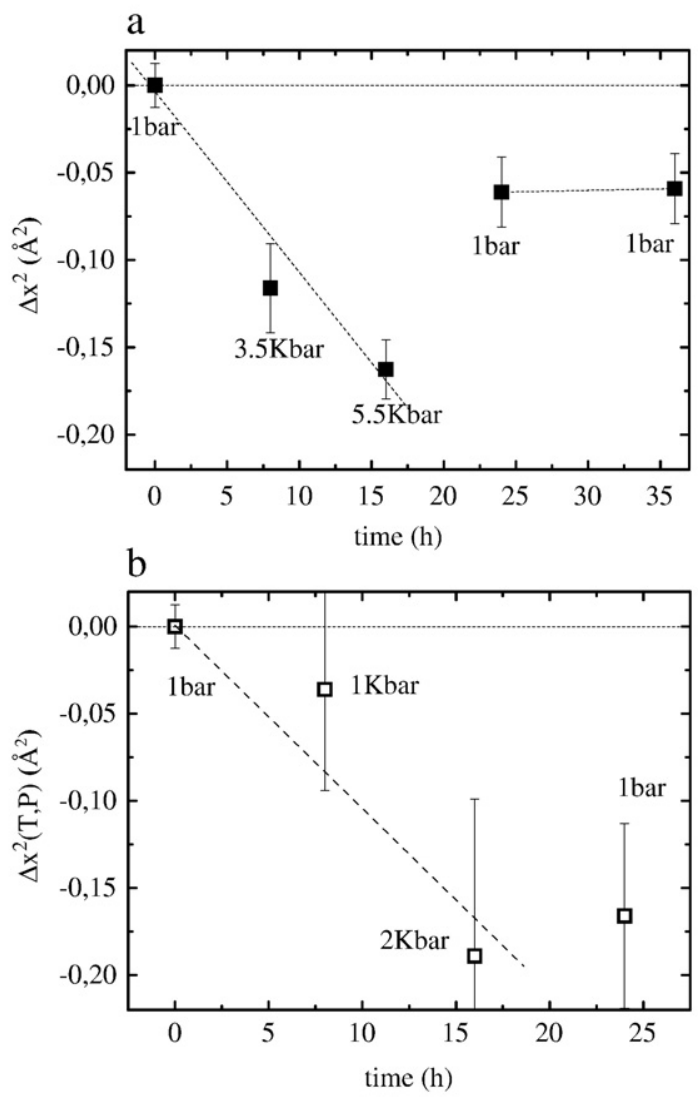

Fig. 5. (a) Time evolution of the difference slope $\Delta x^{2}$, that is $\Delta\left\langle u^{2}(P, T)\right\rangle / 3$, vs pressure for lysozyme + tris buffer at $p=1,3500,5500,1$ bar. The data have been normalized to the reference data set at $P_{0}=1$ bar at $T_{0}=298 \mathrm{~K}$. (b) Time evolution of the difference slope $\Delta x^{2}$, that is $\Delta\left\langle u^{2}(P, T)\right\rangle / 3$, vs pressure for lysozyme + acetate buffer at $p=1,1000$, 2000, 1 bar. The data have been normalized to the reference data set at $P_{0}=1$ bar at $T_{0}=298 \mathrm{~K}$. 


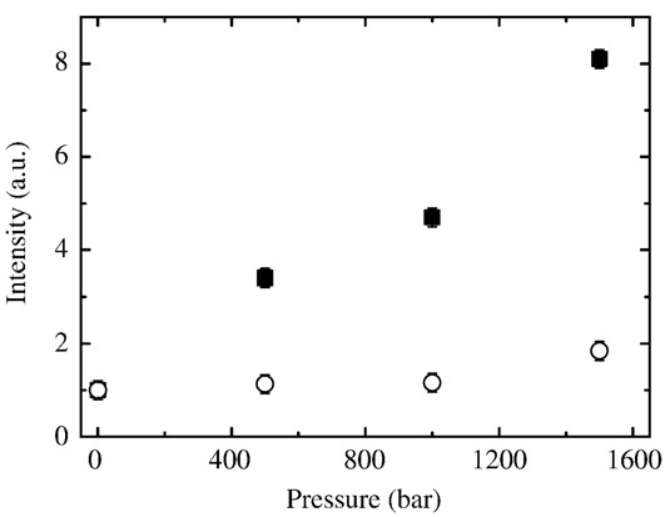

Fig. 6. Fluorescence light scattering intensity of lysozyme in acetate (squares) and tris (circles) buffer as a function of pressure upon excitation at $520 \mathrm{~nm}$. The data are shown after subtraction of the buffer contribution.

the protein. Furthermore, the irreversibility observed upon pressure release to 1 bar and the lack of recovery of the initial MSF value (see Fig. 5) can be related to conformational changes of lysozyme and to the high propensity of this partial unfolded state to form amyloids upon decompression [19], although solvent modifications in the first hydration shell can not be excluded, as it was shown at nondenaturating pressure on lysozyme in solution.

\section{Conclusions}

Elastic incoherent neutron scattering has proven to be a sensitive tool for the investigation of the pressure dependence of protein conformational fluctuations. In high-pressure experiments, where the scattering from the cell is a relevant fraction of the total signal, ENS has some clear advantages. The elastic signal is much stronger than the inelastic and quasielastic ones enabling one to work with solutions in the $10 \%$ concentration range which are relatively close to the ones existing in cells. The contribution of the solvent is totally flat in the energy range probed by ENS, when using high resolution spectrometers ( $\Delta E \leq 10-15 \mu \mathrm{eV})$, making buffer subtraction easy. In addition even if $\mathrm{D}_{2} \mathrm{O}$ buffers are preferable because of a better signal to noise ratio, in principle one can work even with hydrogenous solvents i.e. in a situation closer to that of natural systems. Our results on trypsin, $\beta$-lactoglobulin and lysozyme have shown that differences in the mean square fluctuations, that can be related to different steps in the denaturing processes and to the high propensity of lysozyme to form amyloids, can be evidenced. This makes ENS a useful microscopic technique for the investigation of changes in protein flexibility and resilience induced by high pressure.

\section{Acknowledgements}

The authors gratefully acknowledge the contribution by the CRGIN13 team for the development of the high pressure cell and the support provided during the scattering experiments.
D.R thanks TOP INDUSTRY for collaborative interaction in the manufacturing of the High Pressure Cell used in the neutron scattering experiments. D.R. acknowledges M. Gonzalez and D. Richard from I.L. L. for their great support on improving the LAMP program for IN13 data analysis.

\section{References}

[1] C.M. Dobson, Protein misfolding, evolution and disease, Trends Biochem. Sci. 24 (1999) 329-332.

[2] J. Zhang, P. Xiangdong, A. Jonas, J. Jonas, NMR study of the cold, heat and pressure unfolding of ribonuclease A, Biochem. 34 (1995) 8631-8641.

[3] J.L. Silva, D. Foguel, Hydration, cavities and volume in protein folding, aggregation and amyloid assembly, Phys. Biol. 6 (2009) 15002-15013.

[4] F.L. Palhano, L.P. Leme, R.G. Busnardo, D. Foguel, Trapping the monomer of a nonamyloidogenic variant of transthyretin: exploring its possible use as a therapeutic strategy against transthyretin amyloidogenic diseases, J. Biol. Chem. 284 (2009) 1443-1453.

[5] M. Nishiyama, Y. Kimura, Y. Nishiyama, M. Terazima, Pressure-induced changes in the structure and function of the kinesin-microtubule complex, Biophys. J. 96 (2009) 1142-1150.

[6] C.L. Dias, T. Ala-Nissila, M. Karttunnen, I. Vattulainen, M. Grant, Microscopic mechanism for cold denaturation, Phys. Rev. Lett. 100 (2008) 118001-118004.

[7] W. Doster, R. Gebhardt, High pressure-unfolding of myoglobin studied by dynamic neutron scattering, Chem. Phys. 292 (2003) 383-387.

[8] M. Di Bari, A. Deriu, A. Filabozzi, C. Andreani, A. Di Venere, N. Rosato, Dynamics of trypsin under pressure, Physica B 276 (2000) 510-511.

[9] A. Filabozzi, M. Di Bari, A. Deriu, A. Di Venere, C. Andreani, N. Rosato, Pressure dependence of protein dynamics by elastic and quasielastic neutron scattering, J. Phys. Condens. Matter 17 (2005) S3101-S3109.

[10] L. Meinhold, J.C. Smith, A. Kitao, A.H. Zewail, Picosecond fluctuating protein energy landscape mapped by pressure-temperature molecular dynamics simulation, PNAS 104 (2007) 1761-1765.

[11] V. Calandrini, V. Hamon, K. Hinsen, P. Calligari, M.-C. Bellissent-Funel, G.R. Kneller, Relaxation dynamics of lysozyme in solution under pressure: combining molecular dynamics simulations and quasielastic neutron scattering, Chem. Phys. 345 (2008) 289-297.

[12] M.G. Ortore, A. Paciaroni, F. Spinozzi, P. Mariani, H. Amenitsch, J. Ollivier, L. Barbosa, D. Russo, Combining Structure and dynamics: non denaturing high pressure effect on lysozyme in solution, J. R. Soc. Interface 6 (2009) S619-S634.

[13] G. Zaccai, How soft is a protein? A protein dynamics force constant measured by neutron scattering, Science 288 (2000) 1604-1607.

[14] D.J. Bicout, G. Zaccai, Protein flexibility from the dynamical transition: a force constant analysis, Biophys. J. 80 (2001) 1115-1123.

[15] L. Meinhold, D. Clement, M. Tehei, R. Daniel, J.L. Finney, J.C. Smith, Protein dynamics and stability: the distribution of atomic fluctuations in thermophilic and mesophilic dihydrofolate reductase derived using elastic incoherent neutron scattering, Biophys. J. 94 (2008) 4812-4818.

[16] K. Ruan, R. Lange, N. Bec, C. Balny, A stable partly denatured state of trypsin induced by high hydrostatic pressure, Biochem. Biophys. Res. Commun. 239 (1997) 150-154.

[17] M. Gross, G. Auerbach, R. Jaenicke, The catalytic activities of monomeric enzymes show complex pressure dependence, FEBS Lett. 32 (1993) 256-260.

[18] K. Akasaka, T. Tezuka, H. Yamada, Pressure-induced changes in the folded structure of lysozyme, J. Mol Biol. 271 (1997) 671-678.

[19] F.G. De Felice, M.N. Vieira, M.N. Meirelles, L.A. Morozova-Roche, C.M. Dobson, S.T. Ferreira, Formation of amyloid aggregates from human lysozyme and its diseaseassociated variants using hydrostatic pressure, FASEB J. 18 (2004) 1099-1101.

[20] A.J. Sophianopoulos, K.E. Van Holde, Physical studies of muramidase (Lysozyme). II. pH-dependent dimerisation, J. Biol. Chem. 239 (1964) 2516-2524.

[21] A.A. Paladini, G. Weber, Absolute measurements of fluorescence polarization at high pressure, Rev. Sci. Instrum. 52 (1981) 419-427.

[22] http://www.ill.eu/instruments-support/instruments-groups/instruments/ in13/characteristics/.

[23] LAMP, the Large Array Manipulation Program: http://www.ill.fr/data_treat/ lamp/lamp.html.

[24] S. Kunugi, M. Fukuda, N. Ise, Pressure dependence of trypsin-catalyzed hydrolyses of specific substrates, Biochim. Biophys. Acta 704 (1982) 107-113. 